\title{
¿PANAMÁ EN LA RUTA DEL DESARROLLO SOSTENIBLE?*
}

\author{
Manuel A. Zambrano-Monserrate \\ Maria Alvarado-Sánchez ${ }^{\mathrm{b}}$ \\ María González-Sánchezi \\ Karen Rivas-Ávila \\ Marola Beltrán-Mora
}

* DOI: https://doi.org/10.18601/01245996.v20n38.12. Este artículo es resultado de la investigación sobre Economía Ambiental, Escuela Superior Politécnica del Litoral, Guayaquil, Ecuador. Recepción: 04-04-2016, modificación: 12-01-2018, aceptación: 19-02-2018. Sugerencia de citación: Zambrano-M., M. A.; Alvarado S., M.; González S., M.; Rivas-Á., K. y Beltrán-M., M. (2018). ¿Panamá en la ruta del desarrollo sostenible? Revista de Economía Institucional, 20(38), 285-302.

a Magíster en Economía y Dirección de Empresas. Profesor de la Escuela Superior Politécnica del Litoral, ESPOL, Guayaquil, Ecuador, [anzambra@ espol.edu.ec].

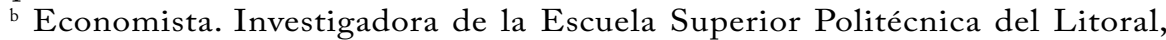
Guayaquil, Ecuador, [maisalva@espol.edu.ec].

' Economista. Investigadora de la Escuela Superior Politécnica del Litoral, Guayaquil, Ecuador, [mfgonzal@espol.edu.ec].

d Economista. Investigadora de la Escuela Superior Politécnica del Litoral, Guayaquil, Ecuador, [kgrivas@espol.edu.ec].

' Magíster en Economía y Dirección de Empresas. Profesora de la Universidad Técnica de Babahoyo, Ecuador, [mbeltranm@utb.edu.ec]. 


\section{¿Panamá en la ruta del desarrollo sostenible?}

Resumen. Este artículo examina la relación de largo plazo entre emisiones de $\mathrm{CO}_{2}$, PIB y consumo de energía en Panamá durante el periodo 1971-2011 mediante un modelo autorregresivo de retardos distribuidos para comprobar la cointegración de las variables en el largo plazo. Los resultados confirman la presencia de una curva ambiental de Kuznets y que las emisiones de $\mathrm{CO}_{2}$ y el consumo de energía de fuentes primarias no afectan al crecimiento económico y que la degradación ambiental aumenta con el consumo de energía. Por último, hace recomendaciones de política.

Palabras claves: energía, ARDL, crecimiento económico, Panamá; JEL: Q56, Q53, C51, C22

\section{Is Panama on a sustainable development path?}

Abstract. This article examines the long-term relationship between $\mathrm{CO}_{2}$ emissions, GDP and energy consumption in Panama during the period 1971-2011 through an autoregressive model of distributed delays to verify the cointegration of variables in the long term. The results confirm the presence of an environmental Kuznets curve and that $\mathrm{CO}_{2}$ emissions and energy consumption from primary sources do not affect economic growth and that environmental degradation increases with energy consumption. Finally, it makes some policy recommendations.

Keywords: energy, ARDL, economic growth, Panamá; JEL: Q56, Q53, C51, C22

\section{¿O Panamá a caminho do desenvolvimento sustentável?}

Resumo. Este artigo examina a relação a longo prazo entre emissões de $\mathrm{CO}_{2}, \mathrm{PIB}$ e consumo de energia no Panamá durante o periodo 1971-2011, mediante um modelo autorregressivo de atrasos distribuídos para comprovar a cointegração das variáveis a longo prazo. Os resultados confirmam a presença de uma curva ambiental de Kuznets e que as emissões de $\mathrm{CO}_{2}$ e o consumo de energia de fontes primárias não afetam o crescimento econômico e que a degradação ambiental é aumentada com o consumo de energia. Por último, faz recomendações políticas.

Palavras-chaves: energia, ARDL, crescimento econômico, Panamá; JEL: Q56, Q53, $\mathrm{C} 51, \mathrm{C} 22$ 
L os países en desarrollo tienden a experimentar tasas de crecimiento económico positivas y crecientes que mejoran los indicadores de desarrollo humano, aunque desmejoran los indicadores ambientales debido a que en las primeras etapas de la industrialización, la contaminación crece rápidamente por la alta prioridad de aumentar la producción de materiales; además, la población está más interesada en emplearse y aumentar sus ingresos que en cuidar el medio ambiente (Dasgupta et al., 2002). En los últimos años un importante tema de estudio ha sido la contraposición entre crecimiento económico y medio ambiente, y se ha encontrado que la contaminación y sus efectos aumentan cuando se busca el máximo ingreso real y disminuyen una vez se alcanza cierto umbral. En suma, que la calidad del medio ambiente se deteriora en la etapa temprana de crecimiento económico y después mejora (Dinda, 2004).

Esta relación se representa mediante la curva ambiental de Kuznets (CAK), que tiene forma de $\mathrm{U}$ invertida y muestra que en las primeras etapas de crecimiento económico la degradación ambiental aumenta, pero más allá de cierto nivel de ingreso la tendencia se invierte: con altos niveles de ingreso el crecimiento económico conduce a una mejora del medio ambiente (Stern, 2004). Si en un país se observa esta relación se dice que está en la ruta del desarrollo sostenible (Zambrano y Fernández, 2017).

A pesar de los numerosos trabajos sobre la relación entre crecimiento económico y contaminación, y entre consumo de energía y crecimiento económico, los resultados de las políticas públicas en diversos países en desarrollo indican que no son adecuadas. Una manera de evaluar esta situación es examinar las variables pertinentes en un marco integrado crecimiento-energía o crecimiento-contaminación (Ang, 2007).

Después de Chile, Panamá es el segundo país latinoamericano de más rápido crecimiento económico en los últimos años. En 2000, cuando suscribió la Declaración del Milenio junto a 192 países, se comprometió a lograr la sostenibilidad ambiental (ONU, 2014). Hoy Panamá promueve seis proyectos de conservación de la biodiversidad, con el apoyo de entidades internacionales y del Banco Mundial. Además, desde 1991 ha experimentado una tasa de crecimiento positiva, y es el país centroamericano de mayor crecimiento económico, con un aumento promedio del PIB superior al 8\% entre 2006 y 2012 (Banco Mundial, 2014). En el Segundo Inventario Nacional de Gases de Efecto Invernadero (SINGEI) se determinó que el $\mathrm{CO}_{2}$ es el principal gas de efecto invernadero (GEI), cuyo principal emisor es el sector energético (ANAM, 2011). 
Para combatir la contaminación ambiental, entre sus objetivos de desarrollo del milenio Panamá incluyó la sostenibilidad ambiental, y ha hecho avances significativos, entre ellos una producción más limpia de energía, proyectos piloto de valoración económica de recursos naturales y cuentas ambientales. Así, a medida que la economía panameña evoluciona se registran avances en proyectos e inversiones ambientales que generan empleo. Esto motiva a examinar la relación entre crecimiento económico y calidad ambiental en el país, pues los aspectos ambientales son cada vez más importantes en las políticas de los diferentes países.

En la primera sección se revisan los trabajos sobre la CAK. En la segunda se describen el modelo y los datos; y en la tercera, la estimación del modelo autorregresivo de retardos distribuidos (ARDL). En la cuarta se discuten los hallazgos empíricos, y en la última se presentan las conclusiones y algunas implicaciones de política.

\section{REVISIÓN DE LA LITERATURA}

La iniciativa de examinar la relación entre renta nacional y medidas de calidad ambiental fue de Grossman y Krueger (1991), quienes luego hicieron la primera prueba empírica de la hipótesis CAK (Grossman y Krueger, 1995). De aquí en adelante se ha publicado resultados de investigaciones que demuestran el aumento de las emisiones de $\mathrm{CO}_{2}$ en la primera etapa del crecimiento económico y su continua reducción después de cierto punto máximo del nivel de ingresos. Al-Mulali y Ozturk (2016) examinan la existencia de la hipótesis de la CAK en 27 economías avanzadas mediante técnicas de panel no estacionarias entre 1990 y 2012. Boutabba (2014) confirma la existencia de una CAK para India y la relación causal a largo plazo entre las emisiones de carbono, el desarrollo financiero, los ingresos, el uso de energía y la apertura comercial. De igual manera, Pao y Tsai (2011) examinan la relación entre emisiones de $\mathrm{CO}_{2}$, consumo de energía y producción entre 2008 y 2013, y encuentran que el daño ambiental y el consumo de energía primero aumentan con mayor ingreso, luego se estabilizan y finalmente disminuyen; además, muestran fuertes relaciones de causalidad bidireccional entre estas tres variables. Iwata et al. (2011) emplean el método de estimación Pooled Mean Group (PMG) para evaluar la hipótesis de la CAK entre emisiones de $\mathrm{CO}_{2}$, PIB y energía nuclear para los países de la OCDE, obteniendo resultados acordes a la teoría establecida.

En los estudios de la CAK se han utilizado diversos contaminantes en el análisis: Hamit-Haggar (2012) utiliza el $\mathrm{CO}_{2}$ para determinar 
la presencia de una CAK en el sector industrial de Canadá entre 1990 y 2007. De forma similar, Zambrano-Monserrate et al. (2016a), Zambrano-Monserrate et al. (2016b), Zambrano-Monserrate et al. (2016c) y Zambrano-Monserrate et al. (2018) emplean en sus estudios el $\mathrm{CO}_{2}$ para validar la hipótesis de la CAK para Brasil, Singapur, Islandia y Perú respectivamente. El óxido de azufre es considerado por Llorca y Meunié (2009) para estimar la CAK en un panel de 28 provincias chinas en el periodo 1996-1999. Así mismo, Flores et al. (2014) y Zambrano-Monserrate y Fernández (2017) emplean el óxido nitroso para comprobar la existencia de una CAK en Estados Unidos y Alemania, respectivamente.

Por otro lado, los factores determinantes de la emisión de estos contaminantes son muy variados, destacando: urbanización (Wang et al., 2016) y energía y comercio (Kasman y Duman, 2015). Además, en estudios realizados para Turquía se determina el uso de energía renovable como variable explicativa de las emisiones de $\mathrm{CO}_{2}$, encontrando como resultado una relación negativa y significativa (Bolük y Mert, 2014; Bolük y Mert, 2015).

Du Can et al. (2015) muestran que existe un aumento de las emisiones de $\mathrm{CO}_{2}$ causado por el uso de energía (producción de electricidad y calor). Según sus resultados, la tasa anual de crecimiento de las emisiones de $\mathrm{CO}_{2}$, entre 2000 y 2010, fue del 3,2\% a nivel mundial. Los autores utilizan una metodología que permite contabilizar las emisiones indirectas de $\mathrm{CO}_{2}$ procedentes del consumo de electricidad y calor en los sectores en los que estos productos energéticos se consumen en última instancia.

Por lo general, los estudios sobre la CAK incluyen otros factores determinantes de la contaminación además del consumo de energía, tales como la urbanización y la apertura comercial. Shahbaz et al. (2014) encuentran una relación positiva entre las emisiones de $\mathrm{CO}_{2}$, el PIB y la urbanización, y una relación negativa entre consumo de energía y nivel de exportaciones, en un estudio realizado para Emiratos Árabes Unidos. Se ha observado también en otros análisis que la urbanización mantiene un efecto positivo sobre las emisiones de $\mathrm{CO}_{2}$; así lo demuestra Sadorsky (2014) en un estudio de 16 economías emergentes durante 1971-2009, donde se estima, por medio de datos de panel con efectos aleatorios, que ante un aumento del 1\% de la urbanización, las emisiones de $\mathrm{CO}_{2}$ se incrementan en un $0,219 \%$. Por otro lado, Farhani et al. (2014) concluyen que un incremento del 1\% en el PIB per cápita aumenta las emisiones de $\mathrm{CO}_{2}$ en un $4,67 \%$ en el largo plazo y en un 6,66\% en el corto plazo; un aumento del $1 \%$ en 
el PIB per cápita al cuadrado disminuye las emisiones de $\mathrm{CO}_{2}$ en un $0,28 \%$ en el largo plazo y en un $1,76 \%$ en el corto plazo; así mismo, un aumento del $1 \%$ en el consumo de energía eleva las emisiones de $\mathrm{CO}_{2}$ en un $0,45 \%$ en el largo plazo y en un $0,70 \%$ en el corto plazo; la variable apertura comercial mantiene efectos positivos sobre las emisiones de $\mathrm{CO}_{2}$ solo en el corto plazo, confirmando así la hipótesis de la CAK para Túnez.

\section{ESPECIFICACIÓN ECONOMÉTRICA}

Se utiliza el marco referencial empleado en Acaravci y Ozturk (2010) para estimar la ecuación de degradación ambiental. La mayoría de los estudios anteriores analizan la causalidad entre crecimiento-contaminación o energía-crecimiento por separado. En otras palabras, se emplean modelos bivariados que presentan el problema de omisión de variables (Shafik y Bandyopadhyay, 1992). Por lo tanto, para evitar este problema, en el estudio se empleó un modelo multivariado, en el cual, además del nivel de emisiones de $\mathrm{CO}_{2}$ y el PIB, se incluyó el consumo de energía, esto es:

$c_{t}=\beta_{0}+\beta_{1} e_{t}+\beta_{2} y_{t}+\beta_{3} y_{t}^{2}+\varepsilon_{t}$

donde $c_{t}$ representa las emisiones de $\mathrm{CO}_{2}$ (dióxido de carbono en toneladas métricas per cápita); $e_{t}$ el consumo de energía (kg equivalente de petróleo per cápita); $y_{t}$ el PIB per cápita a precios constantes de 2005; $y_{t}^{2}$ el cuadrado del PIB per cápita y $\varepsilon_{t}$ el término de perturbación estocástico del modelo. Las letras minúsculas indican la transformación de las variables a logaritmos, lo que permite reducir la heterocedasticidad e interpretar las variables en términos de elasticidad (Ang, 2008). A partir de la gráfica 1 se evidencia que los niveles de PIB per cápita han aumentado con el tiempo, mientras que los niveles de emisiones de $\mathrm{CO}_{2} \mathrm{y}$ de consumo de energía disminuyeron inicialmente, pero aumentaron después de la década de 1990. Los datos para el análisis de las variables son tomados de los Indicadores del Desarrollo Mundial de 2015 para el periodo 1971-2011.

A partir de la revisión de la literatura se espera que el signo de $\beta_{1}$ sea positivo, ya que un mayor nivel de consumo de energía debería dar lugar a una mayor actividad económica, estimulando de esa forma las emisiones de $\mathrm{CO}_{2}$ (Halicioglu, 2009). Además, y a pesar de que la variable energía puede ser medida de distintas maneras, se ha observado que tiene un impacto positivo sobre las emisiones de $\mathrm{CO}_{2}$ (Soytas et al., 2007; Say y Yücel, 2009; Soytas y Sari, 2009; Apergis y 
Payne, 2009). Bajo la hipótesis de la CAK, se espera que la elasticidad de largo plazo de las emisiones de carbono per cápita con respecto al PIB per cápita y al cuadrado del PIB per cápita tenga una relación positiva y negativa, respectivamente (esto es, $\beta_{2}>0$ y $\beta_{3}<0$ ).

\section{Estrategia de estimación}

Diversos enfoques han sido empleados en el análisis del vínculo entre degradación ambiental y crecimiento económico, de forma particular entre las emisiones de $\mathrm{CO}_{2}$, PIB y ciertos factores adicionales causantes de contaminación. Jorgenson y Wilcoxen (1993) y Xepapadeas (2005) modelan la relación entre $\mathrm{CO}_{2}$, PIB y demás contaminantes del medio ambiente utilizando un modelo de crecimiento agregado. Estudios recientes proponen un nuevo enfoque al análisis de la relación entre crecimiento económico-emisiones de $\mathrm{CO}_{2}$ y consumo de energía-emisiones de $\mathrm{CO}_{2}$, incluyendo ambos vínculos en un solo modelo de ecuaciones.

En este se adopta este enfoque y se aplica el modelo autorregresivo de retardos distribuidos (ARDL) desarrollado por Pesarant et al. (2001), debido a sus ventajas frente a otras técnicas de cointegración, como las de Engle y Granger (1987), Johansen y Juselius (1990) y Phillips y Hansen (1990). Primera, su aplicación es independiente del orden de integración de las variables, que pueden ser de orden $0 \mathrm{I}(0)$ o $1 \mathrm{I}(1)$; de modo que el modelo se puede estimar por mínimos cuadrados ordinarios (MCO). Para respaldar esta afirmación Pesarán y Shin (1998) argumentan que, si los estimadores de MCO son consistentes, con este método son aún más consistentes. Segunda, el método ARDL es más preciso para estimar la relación de largo plazo en muestras pequeñas que la técnica de cointegración de Johansen y Juselius (Pesaran y Shin, 1998). Tercera, el modelo de corrección de errores (MCE) tiene suficiente flexibilidad para dar cabida a los rezagos que capturan el proceso de generación de datos en un marco general de especificación (Laurenceson y Chai, 2003). E1 modelo sin restricciones se expresa como sigue:

$\Delta c_{t}=\beta_{0}+\sum_{i=1}^{n} \gamma_{i} \Delta c_{t-1}+\sum_{i=1}^{n} \gamma_{j} \Delta e_{t-j}+\sum_{i=1}^{n} \gamma_{k} \Delta y_{t-k}+\sum_{i=1}^{n} \gamma_{t} \Delta y_{t-1}^{2}+\alpha_{1} c_{t-1}$

$+\alpha_{2} e_{t-1}+\alpha_{3} y_{t-1}+\alpha_{4} y_{t-1}^{2}+U_{t}$

donde $\Delta$ es la primera diferencia, $\beta_{0}$ una constante y $U_{t}$ una variable ruido blanco. La ecuación (2) presenta la dinámica en el corto y en el largo plazo. 
La relación entre el largo plazo entre las variables se mide con los parámetros $\alpha_{1}, \alpha_{2}, \alpha_{3}, \alpha_{4}$. La hipótesis nula de no cointegración en el modelo es $\alpha_{1}=\alpha_{2}=\alpha_{3}=\alpha_{4}=0$.Mientras, la hipótesis alternativa de cointegración es que al menos un alpha sea distinto de cero $\left(\alpha_{k} \neq 0\right)$. Previo a analizar el enfoque de cointegración mediante la prueba de Wald, se debe elegir el mejor modelo ARDL basado en la selección del rezago óptimo, el cual estará en función del criterio de información de Akaike (CIA) y del criterio bayesiano de Schwarz (CBS).

Para realizar el análisis de cointegración de las variables se consideran los valores críticos tabulados por Narayan (2005), ya que estos se calcularon para tamaños de muestras pequeñas, como en el caso de estudio. Los dos conjuntos de valores críticos se presentan para un nivel de significancia dado, con y sin tendencia temporal, uno para variables $\mathrm{I}(0)$ y el otro conjunto $\mathrm{I}(1)$, que se conocen como límites críticos inferiores y superiores, respectivamente. La hipótesis nula de no cointegración se rechaza cuando la F-estadística calculada es más alta que la UCB. En tal situación existe una relación de largo plazo entre las variables. Dado esto, el comportamiento de corto plazo es investigado por el siguiente modelo que incluye el término de corrección de error (TCE):

$\Delta c_{t}=\beta_{0}+\sum_{i=1}^{n} \gamma_{i} \Delta c_{t-1}+\sum_{j=0}^{n} \gamma_{j} \Delta e_{t-j}+\sum_{k=0}^{n} \gamma_{k} \Delta y_{t-k}+\sum_{l=0}^{n} \gamma_{l} \Delta y_{t-l}^{2}+\theta_{1} T C E_{t-1}+U_{t}$

La importancia de incluir el TCE en la ecuación de corto plazo se deriva del hecho de que recoge las desviaciones del equilibrio en el largo plazo de la variable dependiente. Es decir, el TCE asegura que exista convergencia de la variable dependiente al equilibrio de largo plazo, debido a las variaciones en las variables independientes (Shahbaz et al., 2012).

Además de las pruebas de diagnóstico (heterocedasticidad, autocorrelación serial, especificación funcional y normalidad), la ecuación de corto plazo debe asegurar la estabilidad de los parámetros; esto se comprueba por medio de las pruebas de la suma acumulada de los residuos recursivos (CUSUM) y la suma acumulada de los residuos recursivos al cuadrado (CUSUMQ).

Después de comprobar que existe cointegración entre las series, se estima el modelo de corrección de errores vectoriales (VECM, por sus siglas en inglés) para encontrar las relaciones causales entre las variables. El VECM es el enfoque más adecuado para examinar causalidad entre variables cuando las series son integradas de primer orden I(1), como lo son en el presente estudio (Granger, 1969).

La ecuación de causalidad de Granger se define como sigue: 
$\left[\begin{array}{c}\Delta c \\ \Delta y \\ \Delta y^{2} \\ \Delta e\end{array}\right]=\left[\begin{array}{l}\mu_{1} \\ \mu_{2} \\ \mu_{3} \\ \mu_{4}\end{array}\right]+\left[\begin{array}{llll}\varphi_{11, i} & \varphi_{12, i} & \varphi_{13, i} & \varphi_{14, i} \\ \varphi_{21, i} & \varphi_{22, i} & \varphi_{23, i} & \varphi_{24, i} \\ \varphi_{31, i} & \varphi_{32, i} & \varphi_{33, i} & \varphi_{34, i} \\ \varphi_{41, i} & \varphi_{42, i} & \varphi_{43, i} & \varphi_{44, i}\end{array}\right]\left[\begin{array}{c}\Delta c_{t-1} \\ \Delta y_{t-1} \\ \Delta y_{t-1}^{2} \\ \Delta e_{t-1}\end{array}\right]+\left[\begin{array}{c}\omega_{1} \\ \omega_{2} \\ \omega_{3} \\ \omega_{4}\end{array}\right] T C E_{t-1}+\left[\begin{array}{c}\varepsilon_{1 t} \\ \varepsilon_{2 t} \\ \varepsilon_{3 t} \\ \varepsilon_{4 t}\end{array}\right]$

donde el vector de error se distribuye normalmente con media cero y varianza constante. $\mathrm{El}$ vector $\omega$ mide la velocidad del ajuste hacia el equilibrio. Masih y Masih (1997) manifiestan que es posible estimar dos tipos de relación de causalidad de Granger mediante VECM. Si el TCE es significativo, entonces existe una causalidad de Granger a largo plazo; mientras que si las variables explicativas con rezagos en las diferencias influyen significativamente, se dice que existe causalidad de Granger a corto plazo.

\section{RESULTADOS EMPÍRICOS}

Como primer paso, la aplicación de la metodología ARDL requiere demostrar la estacionariedad de las variables para evitar regresiones espurias. En primera instancia, con las gráficas y el correlograma ${ }^{1}$ fue posible evidenciar la presencia de no estacionariedad. Dado que la metodología aplicada asume que las variables son $\mathrm{I}(0)$ o $\mathrm{I}(1)$ (Jalil y Mahmud, 2009), es preciso emplear una prueba formal de raíz unitaria que permita verificar que las variables no sean $\mathrm{I}(2)$. Este estudio utiliza la prueba Dicky-Fuller Aumentada (ADF) para todas las series. La prueba demuestra que las variables $c_{t}, e_{t}, y_{t}$ y $y_{t}^{2}$ son no estacionarias a nivel, ni con tendencia determinista ni estocástica. Sin embargo, resultaron ser estacionarias al obtener su primera diferencia (cuadro 1 ).

Cuadro 1

Prueba de raíz unitaria

\begin{tabular}{lccccc}
\hline & $\mathrm{ADF}$ & $\mathrm{k}$ & & $\mathrm{ADF}$ & $\mathrm{k}$ \\
\hline $\mathrm{c}$ & 0,1552 & 0 & $\Delta \mathrm{c}$ & $7,6414^{* * *}$ & 0 \\
$\mathrm{e}$ & 0,0489 & 0 & $\Delta \mathrm{e}$ & $5,6548^{* * *}$ & 0 \\
$\mathrm{y}$ & 1,6487 & 1 & $\Delta \mathrm{y}$ & $3,3220^{* * *}$ & 0 \\
$\mathrm{y}^{2}$ & 1,6910 & 1 & $\Delta \mathrm{y}^{2}$ & $3,2197^{* * *}$ & 0 \\
Nota: k es la longitud del rezago que se determina de forma automática; & **** significancia al $1 \%$. \\
Fuente: elaboración propia.
\end{tabular}

Una vez definido el orden de integración de las series, el siguiente paso consiste en seleccionar el número óptimo de retardos para incluir en la ecuación (2). Considerando que el valor del F-estadístico es sensible al número de retardos en variables diferenciadas, se tomó como modelo inicial una combinación ARDL $(2,2,2,2)$. Partiendo desde este

${ }^{1}$ Los lectores pueden solicitar a los autores el correlograma de las variables. 
punto se hicieron 36 combinaciones hasta tener el mejor modelo. Se seleccionó el orden óptimo de retardos del modelo basado en el CIA y el CBS, donde el mejor modelo dinámico es el que contiene el valor más bajo de estos criterios (Bölük y Mert, 2015). En este análisis se emplea el modelo (1,0,0,0), que da valores de $-1,2703$ y -0,8864 para el CIA y el CBS, respectivamente (cuadro 2).

Después de comprobar que las variables son integradas de primer orden y seleccionar el mejor modelo $\mathrm{ARDL}^{2}$ el siguiente paso es determinar la presencia de una relación de largo plazo (cointegración). Luego de estimar la regresión por MCO se analiza la cointegración con la prueba de Wald, que proporciona un estadístico $\mathrm{F}=3,561$. Los valores críticos a un nivel de significancia del $10 \%$ son $\mathrm{I}(0)=2,592$; $I(1)=3,454$. De estos resultados se infiere que en el largo plazo hay una relación estable entre emisiones de carbono, consumo de energía y PIB en Panamá ${ }^{3}$.

Cuadro 2

Diferentes combinaciones de modelos ARDL

\begin{tabular}{lcc}
\hline Combinación & CIA & CBS \\
\hline 2201 & $-1,1511$ & $-0,5909$ \\
2110 & $-1,1260$ & $-0,6089$ \\
2101 & $-1,1988$ & $-0,6817$ \\
2010 & $-1,1783$ & $-0,7043$ \\
1210 & $-1,1251$ & $-0,6080$ \\
1201 & $-1,1802$ & $-0,6631$ \\
1021 & $-1,1796$ & $-0,6625$ \\
1012 & $-1,1658$ & $-0,6486$ \\
1002 & $-1,2041$ & $-0,7301$ \\
1000 & $-1,2703$ & $-0,8864$ \\
\hline Nota: CIA = criterio de información de Akaike; CBS = criterio bayesiano de Schwarz. \\
Fuente: elaboración propia.
\end{tabular}

La relación a largo plazo se presenta en el cuadro 3. La variable $e$ muestra un impacto positivo sobre las emisiones de $\mathrm{CO}_{2}$; el coeficiente de 0,8152 es estadísticamente significativo e indica que un incremento del $1 \%$ en el consumo de energía aumentará aproximadamente en $0,82 \%$ las emisiones de $\mathrm{CO}_{2}$ en el largo plazo. Este resultado (elasticidad positiva menor a 1 ) coincide con la relación encontrada por Fuinhas y Marques (2012). De forma similar, el coeficiente 11,6352 es estadísticamente significativo para $y$, y ante

\footnotetext{
${ }^{2}$ Se hicieron todas las pruebas de diagnóstico sobre el mejor modelo ARDL: correlación serial, normalidad, forma funcional y heterocedasticidad, y el modelo pasó todas las pruebas. Los resultados se pueden soilicitar a los autores.

${ }^{3}$ Utilizando el mismo procedimiento se verificó la cointegracion de las variables cuando $y_{t}, y_{t}^{3}$ y $e_{t}$ eran las dependientes. Los resultados se pueden soilicitar a los autores.
} 
un aumento del 1\% del PIB per cápita las emisiones de $\mathrm{CO}_{2}$ crecerán un $11,64 \%$ en el largo plazo. Mientras que la significancia estadística del signo negativo de $y^{2}$ evidencia que el deterioro ambiental decrece para niveles altos de PIB per cápita. El turning point del PIB real per cápita, resultó ser US $\$ 6.450$, el cual resultó menor en comparación con el valor más alto en nuestra muestra, que fue de USD 6.800. E1 resultado confirma que las emisiones de $\mathrm{CO}_{2}$ aumentan en la etapa inicial de crecimiento económico, y disminuyen con el tiempo después de alcanzar el umbral máximo de USD 6.450 correspondiente al PIB.

Se hicieron las siguientes pruebas de diagnóstico, de correlación serial, normalidad, forma funcional y heterocedasticidad. Las estadísticas relevantes se muestran en el cuadro 3. El modelo pasó todas las pruebas.

Cuadro 3

Estimación en el largo plazo

\begin{tabular}{|c|c|c|}
\hline \multicolumn{3}{|l|}{ Variable dependiente: $\mathrm{c}$} \\
\hline Regresores & Coeficientes & Valores-t \\
\hline $\mathrm{E}$ & 0,8152 & $7,3379 * * *$ \\
\hline Y & 11,6352 & $1,8597^{*}$ \\
\hline$y^{2}$ & $-0,6632$ & $-1,7741^{*}$ \\
\hline Intercepto & $-55,7703$ & $-2,1191^{* * *}$ \\
\hline Pruebas de diagnóstico & Valor del estadístico & Valores-p \\
\hline Correlación serial & 1,8141 & 0,1780 \\
\hline Forma funcional & 0,7599 & 0,4523 \\
\hline Normalidad & 3,3518 & 0,1871 \\
\hline Heterocedasticidad & 0,2136 & 0,6466 \\
\hline \multicolumn{3}{|c|}{$\begin{array}{l}\text { Nota: }{ }^{* * *} y^{* * * *} \text { significancia al } 10 \%, 5 \% \text { y } 1 \% \text {, respectivamente. } \\
\text { Fuente: elaboracion propia. }\end{array}$} \\
\hline \multicolumn{3}{|c|}{ Cuadro 4} \\
\hline \multicolumn{3}{|c|}{ Estimación en el corto plazo } \\
\hline \multicolumn{3}{|c|}{ Variable dependiente: $\Delta c$} \\
\hline Regresores & Coeficientes & Valores-t \\
\hline$\overline{\Delta c(-1)}$ & 0,0615 & 0,4213 \\
\hline$\Delta \mathrm{e}$ & 0,8408 & $3,3272^{* * * *}$ \\
\hline$\Delta y$ & 9,9775 & 0,7832 \\
\hline$\Delta y^{2}$ & $-0,5838$ & $-0,7568$ \\
\hline Intercepto & 0,0103 & 0,5023 \\
\hline $\operatorname{TCE}(-1)$ & $-0,7642$ & $-3,8832^{* * *}$ \\
\hline Pruebas de diagnóstico & Valor del estadístico & Valores-p \\
\hline Correlación serial & 0,0715 & 0,9311 \\
\hline Forma funcional & 1,5169 & 0,1391 \\
\hline Normalidad & 4,1529 & 0,2257 \\
\hline Heterocedasticidad & 0,3643 & 0,5499 \\
\hline
\end{tabular}

Nota: ${ }^{* * * *}$ significancia al $10 \%$.

Fuente: elaboración propia. 


\section{Gráfica 1}

\section{Pruebas de CUSUM y CUSUM²}

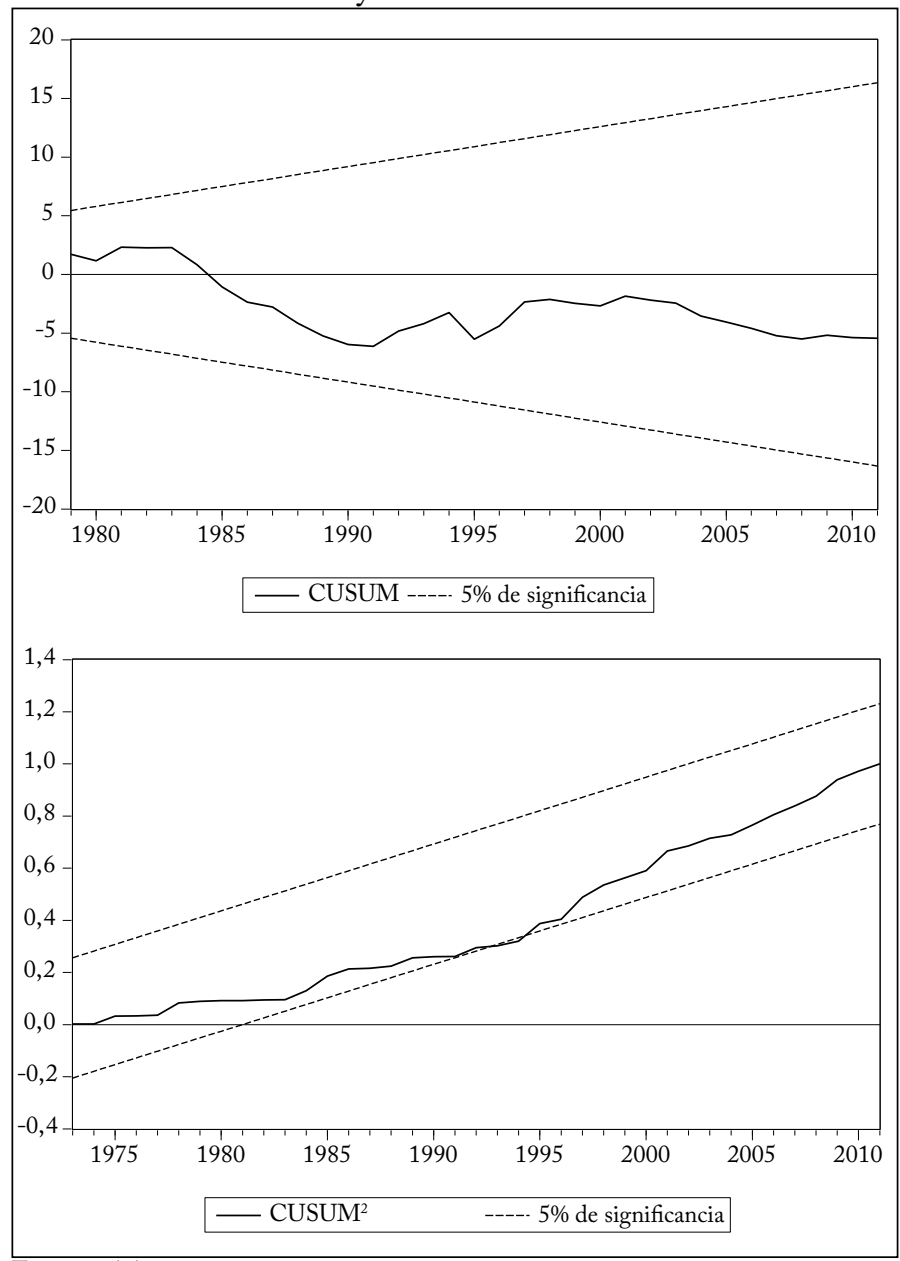

Fuente: elaboración propia.

Los resultados de la dinámica de corto plazo se presentan en el cuadro 4. Los signos obtenidos de las variables explicativas son los esperados. El signo del término de corrección de error TCE es negativo y significativo al 1\%, lo que corrobora la relación de largo plazo establecida entre las variables. El valor rezagado del TCE muestra que las desviaciones del equilibrio en las emisiones de $\mathrm{CO}_{2}$ son corregidas en un $76,42 \%$ dentro de un año. Como se aprecia en la gráfica 1, los parámetros son estables en el periodo 1971-2011.

La metodología ARDL no establece si $y_{t}$ causa a $c_{t}$, si $c_{t}$ causa a $y_{t}$ o si, por el contrario, existe causalidad bidireccional. Por esta razón, 
este estudio también explora la dirección de la relación causal entre las variables, estimando un VECM, el cual es adecuado cuando las variables son integradas de orden 1 (Zambrano et al., 2016a; Saboori y Sulaiman, 2013). Es así que la relación causal entre las variables se analizó mediante la prueba de causalidad de Granger tanto para el corto como para el largo plazo.

Los resultados reportados en el cuadro 5 muestran que el coeficiente de TCE (-1) es significativo con signo negativo en la ecuación de emisiones de $\mathrm{CO}_{2}$ e implica que el choque expuesto por el sistema converge al equilibrio de largo plazo a una velocidad del 96,7\%. De otra parte, es posible sugerir que en Panamá la degradación del medio ambiente no tiene un impacto causal en el crecimiento económico. Las conclusiones de las pruebas de causalidad de Granger reportadas en el cuadro 6 sugieren que existe un impacto causal unidireccional que va desde los niveles de consumo de energía y PIB hacia las emisiones de $\mathrm{CO}_{2}$. En el mismo sentido, Ahmad et al. (2017) encuentran una causalidad unidireccional que va desde el PIB a las emisiones de $\mathrm{CO}_{2}$ en Corea del Sur.

Cuadro 5

Prueba de causalidad de Granger

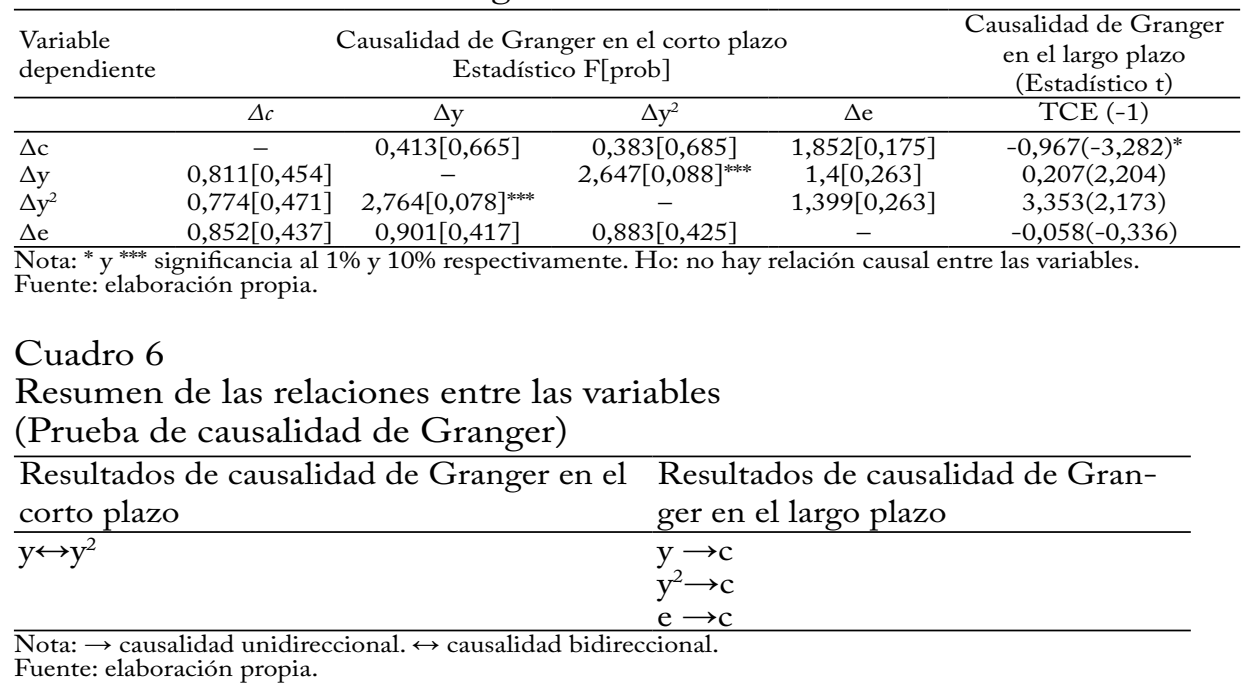

\section{CONCLUSIONES}

Este artículo examina por vez primera la CAK de Panamá en 19712011. Los principales resultados confirman la presencia de una CAK a largo plazo, lo que indica que está en la ruta del desarrollo sostenible. 
La explicación más acertada de la relación positiva entre PIB y emisiones de $\mathrm{CO}_{2}$, y negativa entre el cuadrado del PIB y emisiones de $\mathrm{CO}_{2}$, se refleja en la elasticidad ingreso de la demanda de calidad del medio ambiente (McConnell, 1997). A mayores ingresos, las personas no solo están dispuestas a pagar más por productos positivos para su salud, sino también a ejercer más presión para que se proteja el medio ambiente y se cumplan las normas. En la mayoría de los casos donde las emisiones han disminuido con el aumento de los ingresos, las reducciones se deben a reformas institucionales locales y nacionales, como la legislación ambiental y los incentivos de mercado para reducir la degradación ambiental (Dinda, 2004).

La prueba de causalidad de Granger permite analizar las implicaciones de política. Los resultados sugieren que las políticas para reducir el $\mathrm{CO}_{2}$ no desaceleran el desempeño económico de largo plazo en Panamá ni que una mayor inversión en opciones que reduzcan la contaminación tenga consecuencias en el crecimiento económico. En cambio, la expansión de la economía panameña ejerce un efecto causal positivo de reducir las emisiones contaminantes en el largo plazo. Por tanto, en materia ambiental es necesario adoptar políticas sobre los componentes de la producción. Seker et al. (2015) encuentran que la inversión extranjera directa (IED) tiene un efecto causal significativo en el crecimiento económico; en consecuencia, se debería fomentar la entrada de IED en industrias que empleen tecnología intensiva con energías limpias, una política ambientalmente factible y económicamente viable en Panamá.

Por otro lado, la falta de causalidad entre el uso de energía y el PIB implica que la economía panameña no es vulnerable a cambios en el sector energético primario; sin embargo, los esfuerzos para lograr la eficiencia energética en este sector generan una externalidad positiva al reducir los niveles de emisión de $\mathrm{CO}_{2}$. Se sugiere entonces que se impulsen proyectos de eficiencia energética en el sector de transportes, que consume el $56 \%$ de los derivados de petróleo a nivel nacional y es el principal consumidor de energía.

\section{REFERENCIAS BIBLIOGRÁFICAS}

Acaravci, A. y Ozturk, I. (2010). On the relationship between energy consumption, $\mathrm{CO}_{2}$ emissions and economic growth in Europe. Energy, 35(12), 5412-5420.

Ahmad, N., Du, L., Lu, J. et al. (2017). Modelling the $\mathrm{CO}_{2}$ emissions and economic growth in Croatia: Is there any environmental Kuznets curve? Energy, 123(15), 164-172. 
A1-Mulali, U. y Ozturk, I. (2016). The investigation of environmental Kuznets curve hypothesis in the advanced economies: The role of energy prices. Renewable and Sustainable Energy Reviews, 54, 16221631.

Ang, J. B. (2007). $\mathrm{CO}_{2}$ emissions, energy consumption, and output in France. Energy Policy, 35(10), 4772-4778.

Ang, J. B. (2008). Economic development, pollutant emissions and energy consumption in Malaysia. Journal of Policy Modeling, 3O(2), 271-278.

Apergis, N. y Payne, J. E. (2009). $\mathrm{CO}_{2}$ emissions, energy usage, and output in Central America. Energy Policy, 37(8), 3282-3286.

Autoridad Nacional del Ambiente. (2011). Segunda Comunicación Nacional ante la Convención Marco de las Naciones Unidas sobre Cambio Climático, Panamá: ANAM.

Banco Mundial (2014). Panorama general 2014. Panamá: BM.

Bolük, G. y Mert, M. (2014). Fossil and renewable energy consumption, GHGs and economic growth: Evidence from a panel of European Union (EU) countries. Energy, 74(5), 439-446.

Bölük, G. y Mert, M. (2015). The renewable energy, growth and environmental Kuznets curve in Turkey: An ARDL approach. Renewable and Sustainable Energy Reviews, 52, 587-595.

Boutabba, M. A. (2014). The impact of financial development, income, energy and trade on carbon emissions: Evidence from the Indian economy. Economic Modelling, 40, 33-41.

Dasgupta, S., Laplante, B., Wang, H. y Wheeler, D. (2002). Confronting the environmental Kuznets curve. The Journal of Economic Perspectives, 16(1), 147-168.

Dinda, S. (2004). Environmental Kuznets curve hypothesis: A survey. Ecological Economics, 49(4), 431-455.

Du Can, S. de la R., Price, L. y Zwickel, T. (2015). Understanding the full climate change impact of energy consumption and mitigation at the end-use level: A proposed methodology for allocating indirect carbon dioxide emissions. Applied Energy, 159, 548-559.

Engle, R. y Granger, C. (1987). Cointegration and error correction representation: Estimation and testing. Econometrica, 55(2), 251-276.

Farhani, S., Mrizak, S., Chaibi, A. et al. (2014). The environmental Kuznets curve and sustainability: A panel data analysis. Energy Policy, 71, 189-198.

Flores, C. A., Flores-L., A. y Kapetanakis, D. (2014). Lessons from quantile panel estimation of the environmental Kuznets curve. Econometric Reviews, 33(8), 815-853.

Fuinhas, J. A. y Marques, A. C. (2012). Energy consumption and economic growth nexus in Portugal, Italy, Greece, Spain and Turkey: An ARDL bounds test approach, 1965-2009. Energy Economics, 34(2), 511-517.

Granger, C. (1969). Investigating causal relations by econometric models and cross-spectral methods. Econometrica: Journal of the Econometric Society, 37(3), 424-438.

Grossman, G. M. y Krueger, A. B. (1991). Environmental impacts of a North American free trade agreement. NBER working paper 3914. 
Grossman, G. M. y Krueger, A. B. (1995). Economic growth and the environment. Quarterly Journal of Economics, 110(2), 353-377.

Halicioglu, F. (2009). An econometric study of CO2 emissions, energy consumption, income and foreign trade in Turkey. Energy Policy, 37(3), 1156-1164.

Hamit-H., M. (2012). Greenhouse gas emissions, energy consumption and economic growth: A panel cointegration analysis from Canadian industrial sector perspective. Energy Economics, 34(1), 358-364.

Iwata, H., Okada, K. y Samreth, S. (2011). A note on the environmental Kuznets curve for CO2: A pooled mean group approach. Applied Energy, 88(5), 1986-1996.

Jalil, A. y Mahmud, S. F. (2009). Environment Kuznets curve for CO2 emissions: A cointegration analysis for China. Energy Policy, 37(12), 5167-5172.

Johansen, S. y Juselius, K. (1990). Maximum likelihood estimation and inference on cointegration - with applications to the demand for money. Oxford Bulletin of Economics and Statistics, 52(2), 169-210.

Jorgenson, D. W. y Wilcoxen, P. J. (1993). Reducing US carbon dioxide emissions: An assessment of different instruments. Journal of Policy Modeling, 15(5), 491-520.

Kasman, A. y Duman, Y. S. (2015). CO 2 emissions, economic growth, energy consumption, trade and urbanization in new EU member and candidate countries: A panel data analysis. Economic Modelling, 44, 97-103.

Laurenceson, J. y Chai, C. H. (2003). Financial reform and economic development in China. Londres: Edward Elgar.

Llorca, M. y Meunié, A. (2009). $\mathrm{SO}_{2}$ emissions and the environmental Kuznets curve: The case of Chinese provinces. Journal of Chinese Economic and Business Studies, 7(1), 1-16.

Masih, A. M. y Masih, R. (1997). On temporal causal relationship between energy consumption, real income and prices: Some new evidence from Asian energy dependent NICs based on a multivariate cointegration vector error correction approach. Journal of Policy Modeling, 19(4), 417-440.

McConnell, K. E. (1997). Income and the demand for environmental quality. Environment and Development Economics, 2(4), 383-399.

Narayan, P. K. (2005). The saving and investment nexus for China: Evidence from cointegration tests. Applied Economics, 37(17), 1979-1990.

ONU (2014). Objetivos de desarrollo del milenio, Cuarto Informe de Panamá.

Pao, H. T. y Tsai, C. M. (2011). Modeling and forecasting the CO2 emissions, energy consumption, and economic growth in Brazil. Energy, 36(5), 2450-2458.

Pesaran, M. H. y Shin, Y. (1998). An autoregressive distributed-lag modelling approach to cointegration analysis. Econometric Society Monographs, 31, 371-413.

Pesaran, M. H., Shin, Y. y Smith, R. J. (2001). Bounds testing approaches to the analysis of level relationships. Journal of Applied Econometrics, 16(3), 289-326. 
Phillips, P. C. y Hansen, B. E. (1990). Statistical inference in instrumental variables regression with $\mathrm{I}(1)$ processes. The Review of Economic Studies, 57(1), 99-125.

Saboori, B. y Sulaiman, J. (2013). Environmental degradation, economic growth and energy consumption: Evidence of the environmental Kuznets curve in Malaysia. Energy Policy, 60, 892-905.

Sadorsky, P. (2014). The effect of urbanization on $\mathrm{CO}_{2}$ emissions in emerging economies. Energy Economics, 41, 147-153.

Say, N. P. y Yücel, M. (2006). Energy consumption and $\mathrm{CO}_{2}$ emissions in Turkey: Empirical analysis and future projection based on an economic growth. Energy Policy, 34(18), 3870-3876.

Seker, F., Ertugrul, H. M. y Cetin, M. (2015). The impact of foreign direct investment on environmental quality: A bounds testing and causality analysis for Turkey. Renewable and Sustainable Energy Reviews, 52, 347-356.

Shafik, N. y Bandyopadhyay, S. (1992). Economic growth and environmental quality: Time-series and cross-country evidence, 904. Washington DC: World Bank.

Shahbaz, M., Lean, H. H. y Shabbir, M. S. (2012). Environmental Kuznets curve hypothesis in Pakistan: Cointegration and Granger causality. Renewable and Sustainable Energy Reviews, 16(5), 2947-2953.

Shahbaz, M., Sbia, R., Hamdi, H. et al. (2014). Economic growth, electricity consumption, urbanization and environmental degradation relationship in United Arab Emirates. Ecological Indicators, 45, 622-631.

Soytas, U. y Sari, R. (2009). Energy consumption, economic growth, and carbon emissions: Challenges faced by an EU candidate member. Ecological Economics, 68(6), 1667-1675.

Soytas, U., Sari, R. y Ewing, B. T. (2007). Energy consumption, income, and carbon emissions in the United States. Ecological Economics, 62(3), 482-489.

Stern, D. I. (2004). The rise and fall of the environmental Kuznets curve. World Development, 32(8), 1419-1439.

Wang, Q., Zeng, Y. E. y Wu, B. W. (2016). Exploring the relationship between urbanization, energy consumption, and $\mathrm{CO}_{2}$ emissions in different provinces of China. Renewable and Sustainable Energy Reviews, 54, 1563-1579.

Xepapadeas, A. (2005). Regulation and evolution of compliance in common pool resources. The Scandinavian Journal of Economics, 107(3), 583-599.

Zambrano-M., M. A., Silva-Z., C. A., Davalos-P. et al. (2018). Testing environmental Kuznets curve hypothesis in Peru: The role of renewable electricity, petroleum and dry natural gas. Renewable and Sustainable Energy Reviews, 82, 4170-4178.

Zambrano-M., M. A., Troccoly-Q., A. y Pacheco-B., M. J. (2016c). Testing the environmental Kuznets curve hypothesis in Iceland: 1960-2010. Revista de Economia del Rosario, 19(1), 5-28.

Zambrano-M., M. A., Valverde-B., I., Aguilar-B., J. et al. (2016a). Relationship between economic growth and environmental degradation: Is there evidence of an environmental Kuznets curve for Brazil? International Journal of Energy Economics and Policy, 6(2), pp. 208-216. 
Zambrano-M., M. A., Carvajal-Lara, C. y Urgiles-S., R. (2016b). Is there an inverted U-shaped curve? Empirical analysis of the environmental Kuznets curve in Singapore. Asia-Pacific Journal of Accounting Eo Economics, 23(4), 1-18.

Zambrano-M., M. A. y Fernández, M. A. (2017). An Environmental Kuznets Curve for $\mathrm{N}_{2} \mathrm{O}$ emissions in Germany: An ARDL approach. Natural Resources Forum, 41, 119-127. 